Dom. Cien., ISSN: 2477-8818

Vol. 5, núm.1., ene, 2019, pp. 299-325

Programación lineal y software para cobertura aplicado a la instalación del servicio de internet en la empresa Solintel S.A

\title{
Programación lineal y software para cobertura aplicado a la instalación del servicio de internet en la empresa Solintel S.A
}

\section{Linear programming and software for coverage applied to the installation of the internet service in Solintel S.A}

\section{Programação linear e software para cobertura aplicada à instalação do serviço de internet na Solintel S.A}

\author{
Dennis H. Zambrano-Silva ${ }^{\mathrm{I}}$ \\ dennis.zambranos@ug.edu.ec \\ Luis E. Arguello-Cortez II \\ luis.arguelloc@ug.edu.ec \\ Javier Domínguez De La Torre ${ }^{\text {III }}$ \\ javier.dominguezd@ug.edu.ec \\ Eladio Bautista-Chalar IV \\ eladio.bautistac@ug.edu.ec
}

Recibido: 27 de junio de 2018 * Corregido: 28 de agosto de 2018 * Aceptado: 20 de septiembre de 2018

\footnotetext{
Magister en Sistemas de Producción y Productividad, Ingeniero Industrial, Universidad de Guayaquil, Ecuador. Ingeniero Industrial, Universidad de Guayaquil, Ecuador.

Magister en Diseño Curricular; Ingeniero Industrial, Universidad de Guayaquil, Ecuador.

IV. Magister en Diseño Curricular; Ingeniero Industrial; Licenciado en Ciencias de la Educación Especialización Informática; Tecnólogo Pedagógico en Informática, Universidad de Guayaquil, Ecuador.
} 


\section{Resumen}

El objetivo de este artículo es resolver por medio de programación lineal la asignación de recursos que permitan optimizar la instalación de los dos tipos de servicios de internet que se proponen ajustándose a los presupuestos en cada zona. Se agrega el uso de un software de cobertura para identificar las zonas que cuentan con poco o ningún servicio de internet. Se establece un posible requerimiento de clientes potenciales, para en lo posterior evaluar las zonas por el número de postes libres y el porcentaje de servicio que se desea instalar en cada zona determinando el costo de instalación total y el número de dispositivos necesarios en cada zona. Como resultado de este trabajo se comprueba que el uso de la programación lineal junto a un software de cobertura facilita la asignación de recursos para la instalación de equipos que proveen el servicio de internet.

Palabras claves: Programación Lineal; Optimización; Cobertura. 


\section{Abstract}

The objective of this article is to solve by means of linear programming the allocation of resources that allow to optimize the installation of the two types of internet services that are proposed adjusting to the budgets in each zone. The use of a coverage software is added to identify areas that have little or no internet service. A possible requirement of potential clients is established, in order to later evaluate the zones by the number of free poles and the percentage of service that it is desired to install in each zone, determining the total installation cost and the number of necessary devices in each zone. As a result of this work it is verified that the use of linear programming together with a coverage software facilitates the allocation of resources for the installation of equipment that provides the internet service.

Key words: Linear Programming; Pymes; Optimization; Coverage. 


\section{Introducción.}

La tecnología en la actualidad es un recurso necesario que ha permitido establecerse de manera fija y segura, además de resolver problemas en diferentes áreas como en la educación, la ciencia entre otras. (Evans, 2011)

La evolución del internet como uno de los dispositivos que permiten una conexión a la red de manera rápida y continua obliga a que grupos o personas de diferentes especializaciones se interesen más en el tema tecnológico como son las redes y dispositivos intermediarios. (Huilcamaigua, 2016).

Los distintos Proveedores de servicio de Internet (ISP) son los que se encargan de brindar conexión a internet a sus clientes con la finalidad de un acceso al medio mediante dispositivos de red, (Raña K, Ferrer O, \& Bedregal G, 2007) en el caso de brindar varios servicios es necesario realizar evaluaciones en cada tipo de servicio para conocer su funcionamiento y manipulación de mismo adecuadamente.

Uno de los campos de investigación en los que se puede aplicar el análisis matemático junto con las consideraciones del servicio tecnológico es el de las comunicaciones, para ello se plantea un problema de aplicación basado en la investigación de operaciones el cual mediante el uso de uno o varios modelos matemáticos es posible minimizar los costos en lo que respecta a los ingresos generados por la provisión de servicio. (Programming et al., 2009).

El objetivo o finalidad es obtener un resultado óptimo que permitirá maximizar la compra de varios dispositivos de red, debido a que en la actualidad se ha notado de manera considerable una demanda por recursos de internet (Kalenatic, 2009).

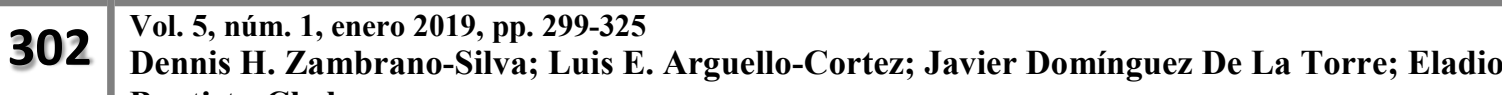
Bautista-Chalar
} 
La presente investigación busca mejorar la asignación de recursos mediante programación lineal en la gestión PYMES (Programming, Programming, Value, \& Prices, 2009), agregando un software de uso libre para posicionamiento y actualización de información.

A continuación, se explican brevemente los conceptos principales acerca del modelo matemático a usar.

Programación Lineal: Procedimiento o algoritmo matemático en el cual se puede resolver un problema que exista, el cual es planteado mediante ecuaciones lineales permitiendo la optimización de la función objetivo. (Ospina, Rodas, \& Botero, 2008).

En el resultado de la programación lineal se incluyen las ecuaciones que más aportan en la descripción de cómo funciona el modelo de instalación del servicio. Una parte fundamental de la programación lineal dentro de la planificación es el método en el cual se utilizan las técnicas matemáticas correspondientes las cuales permiten asignar una modelo como tal (Guédez Fernández, 2011).

Como objeto de estudio nos enfocaremos en la empresa Solintel S.A la misma que está encargada en la distribución del servicio de internet, en la ciudad de Esmeraldas, brindando soluciones en el ámbito de las telecomunicaciones, como también en la informática y electrónica. La distribución del servicio de internet es realizada utilizando los recursos logísticos de la Cía. de estudio, para lo cual el administrador técnico asigna personal, equipos y materiales para realizar la instalación previamente planificada. La reparación y el mantenimiento se efectúan por el mismo personal dentro del área de cobertura asignada.


Bautista-Chalar
} 
El planteamiento del modelo lineal nace como preocupación por explicar la carencia o necesidad actual que existe en los proveedores de internet (ISP) con la finalidad de dar a conocer el funcionamiento correcto que requieren los dispositivos intermediarios (Comunicaci et al., 2016).

Modelo Actual. - La asignación de los trabajos de instalación, se realiza entregando las solicitudes de los clientes según pedido del área de Ventas, por tanto un mismo equipo de trabajo debe recorrer distancias considerables entre una instalación y otra. En caso de una nueva instalación para un cliente existen dos opciones:

a) Utilizar los equipos ya ubicados en instalaciones previas

b) Realizar una instalación completa por falta de clientes anteriores en el área.

Los costos varían de acuerdo a su instalación ya que la empresa tiene equipos con fibra óptica y equipos con cableado UTP que cuestan $\$ 100$ y $\$ 68$ respectivamente, a estos valores se les añade el transporte o movilización al realizar las debidas instalaciones que tienen un costo de $\$ 5$ para dispositivos con cableado UTP y \$20 para dispositivos con cableado de Fibra Óptica. La empresa para brindar soluciones de red a puesto a disposición 1520 dispositivos de red para optimizar sus recursos y cobertura de los mismos en todos los sectores de la ciudad de Esmeraldas donde no abarque un servicio como tal ocasionando un déficit a lo que corresponde el servicio de internet, el horario de atención que se brindara para dar soluciones e instalaciones a grupos de clientes y empresas es de lunes a viernes de $8 \mathrm{~h} 00-16 \mathrm{~h} 00$.

En el área de interés se encuentran 375 postes distribuidos en el área sin cobertura para la instalación de sus equipos de red, los cuales tienen una capacidad máxima de 7 usuarios conectados 
en un mismo cajetín, el personal de la empresa tiene un tiempo determinado de instalación del servicio en el área de cobertura en un máximo de 3 meses.

En relación a una zona en particular, el \# esperado de instalaciones por poste con los dispositivos de red mediante cableado UTP o Fibra Óptica se establecen como política de la empresa, es de al menos un $15 \%$ de los cliente potenciales del sector. Lo cual justificaría un nuevo punto de instalación ya que de no cumplir este porcentaje mínimo se incurrirá en el uso de más recursos o más tiempo del requerido. En este caso se omite la cantidad de pedidos.

Los requerimientos del tipo de instalación UTP o de fibra óptica estimado por la empresa según el área de la ciudad y el tipo de cliente residencial o comercial-industrial es el siguiente:

\title{
Políticas de instalación de dispositivos con cableado UTP y Fibra Optima por zona
}

\author{
$\checkmark \quad$ Zona $1=5$ UTP y 2 Fibra Óptica \\ $\checkmark \quad$ Zona $2=5$ UTP y 2 Fibra Óptica \\ $\checkmark \quad$ Zona $3=4$ UTP y 3 Fibra Óptica \\ $\checkmark \quad$ Zona $4=6$ UTP y 1 Fibra Óptica \\ $\checkmark \quad$ Zona $5=6$ UTP y 1 Fibra Óptica
}

El esquema de trabajo anterior busca reducir los gastos de instalación y equipos empleados, asignando un \% preestablecido al tipo de instalación, según el sector de la Ciudad tenga mayor número de clientes de fibra óptica o servicio UTP. Según registro de la empresa hasta un $14 \%$ de los clientes se quedan sin servicio a pesar de requerirlo. Adicionalmente este tipo de entrega aleatorio 
es frecuente que los sectores de servicio puedan traslaparse por los equipos de instalación. Algunos de los aspectos que pueden ser optimizados de la actual manera de laborar en Solintel S.A. son:

- $\quad$ La eficiencia en la programación del servicio.

- Minimizar los costos de inversión en equipos.

- $\quad$ Traslape en área de atención.

- $\quad$ Asignación de los suministros para la instalación de nuevo cliente.

\section{Metodología.}

Se plantea la realización de un modelo matemático en el cual mediante el uso de las herramientas de programación lineal y software necesarios optimiza la utilización de los recursos para cubrir las áreas que carecen de servicio. (Calderón, Ocampo, \& Echeverry, 2012).

Cabe mencionar que para un mejor análisis de resultados con respecto a rendimiento en la cobertura de los dispositivos intermediarios se utiliza la programación lineal junto a un software de cobertura, lo que nos permitirá ubicar los puntos ciegos que existan dentro del cantón Esmeraldas con respecto al servicio de internet y proporcionará maximización de recursos para la obtención del servicio en los sitios donde no existe cobertura.

El área sin cobertura se divide en 5 zonas o sectores por consideraciones puramente La ciudad se ha dividido en 5 áreas o sectores específicos con la ayuda del software de cobertura Acrylic Wifi donde el departamento técnico de la empresa está encargado de realizar ese tipo de trabajos, se asignan 9 personas que se dividen en 2 grupos, las cuales son: Instalación y

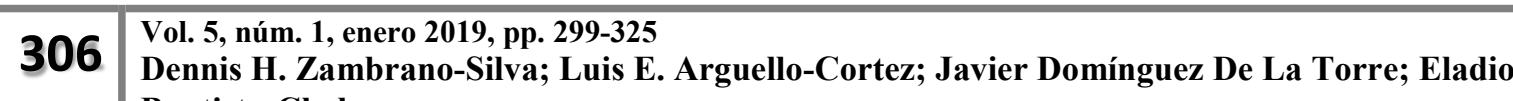


Mantenimiento de los dispositivos de red. En cada sector se realizan instalaciones para hogares y empresas según su ubicación en el área de cobertura asignada. A continuación, en la siguiente tabla se demostrarán los datos antes mencionados.

\section{Tabla 1}

\begin{tabular}{|l|l|}
\hline \multicolumn{2}{|l|}{ PERSONAL TÉCNICO } \\
\hline Instalación en hogares & 2 \\
\hline Instalación en empresas & 3 \\
\hline Personal de Mantenimiento & 4 \\
\hline Total & 9 \\
\hline
\end{tabular}

Fuente: Propia

\section{Elaborado por: Autores}

\section{Relación del modelo de programación lineal aplicado en solver con el análisis de resultados mediante software de cobertura - ACRYLIC HEAT MAPS}

Uno de los factores que afecta a la empresa es la carencia de dispositivos intermediarios o de red en ciertos sitios de la ciudad de Esmeraldas, para ello se optó por usar un modelo lineal realizado en Solver Excel para una maximización de los recursos, adicionalmente se implementó el software, ACRYLIC WIFI HEATMAPS que funciona en tiempo real y con GPS haciendo que la búsqueda de red sea más factible y rápida para encontrar áreas con déficit de cobertura. El software tiene una distribución de forma gratuita por tiempo limitado el mismo que tendrá un costo de licencia. \} 


\section{Ventajas del Software de Cobertura ACRYLIC.}

$\checkmark \quad$ Trabaja mediante planos o croquis de los sitios requeridos.

$\checkmark \quad$ Fácil manejo.

$\checkmark \quad$ Ubicación de Puntos de Acceso o de red.

$\checkmark \quad$ Informe de Cobertura - Ahorro de Recursos

Grafica 1: Verificación de Dispositivos Intermediarios y Carencia de Cobertura Mediante

\section{ACRYLIC HEATMAPS En el Cantón Esmeraldas}

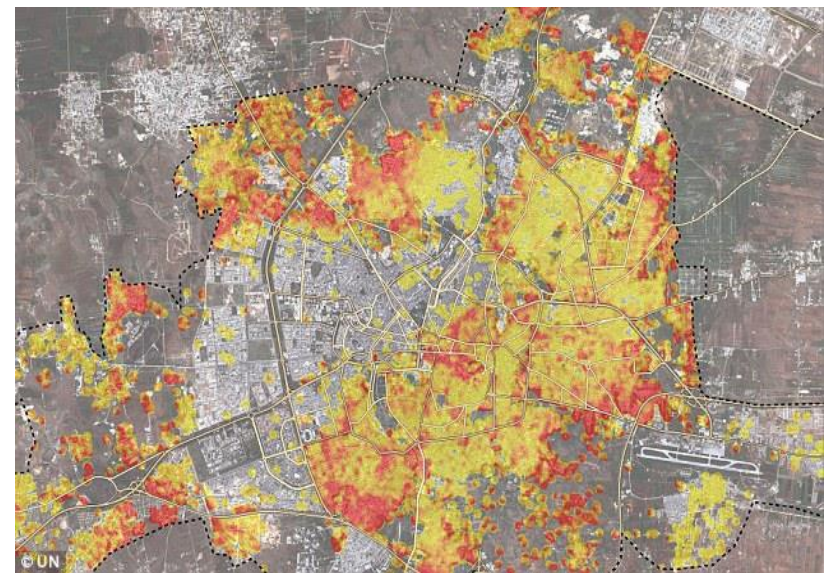

Fuente: Solintel S.A

Elaborado por: Solintel S.A

Como se observa en la Grafica 1 notamos que mediante un Mapeo general realizado en Acrylic Wifi Heat Maps en el Cantón Esmeraldas de la provincia de Esmeraldas se llegó a saber cuáles son los puntos donde se tiene poca o nula cobertura, impidiendo que los usuarios tengan una accesibilidad a la Internet.

Cabe mencionar que las ondas de calor que se producen se deben a la potencia o intensidad con la que cuenta uno o varios dispositivos que están distribuidos en hogares o empresas se aprecian 
en colores determinados para poder determinar su potencia e intensidad de conectividad la cual es medida en Decibelios (-Db) indicando que entre mejor sea la señal de la conectividad en la red se subdividide por colores la potencia de la red como se describe a continuación.

\section{Grafica 2: Areas a Maximizar con Carencia de Cobertura}

\begin{tabular}{|c|c|}
\hline Excelente & \\
\hline Buena & \\
\hline Regular & \\
\hline Nula & \\
\hline
\end{tabular}

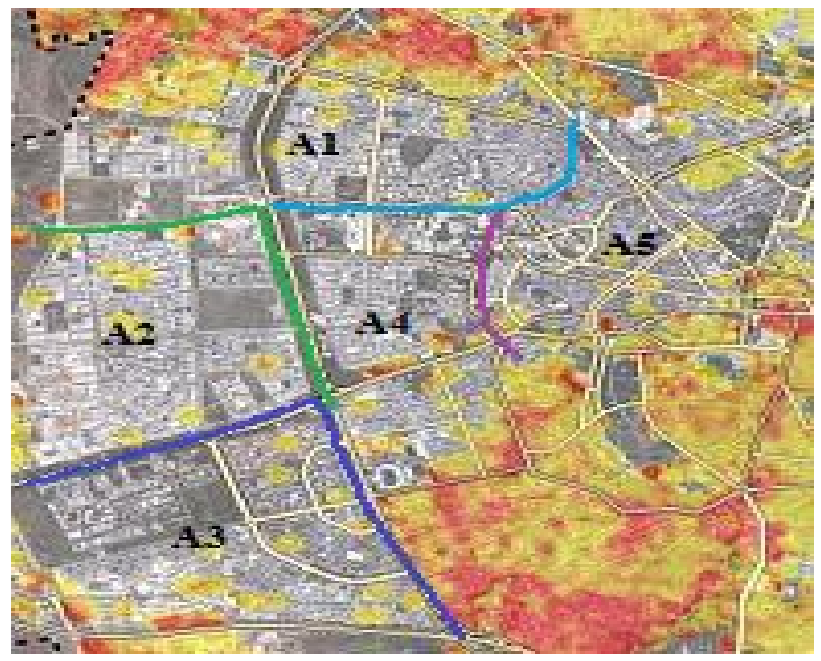

Fuente: Propia

Elaborado por: Autores

\section{Planteamiento del modelo de programación lineal}

\section{Modelamiento de los datos}

Para que un modelo sea posible de solucionarse se requiere la asignación de variables que se vayan a utilizar con su respectiva nomenclatura que se describen a continuación. 
- $\quad \mathrm{X} 1=$ Instalación de Equipos mediante Cable UTP

- $\quad \mathrm{X} 2=$ Instalación de Equipos mediante Fibra Óptica

Según (Redes et al., n.d.) es necesario considerar las variables del modelo de estudio, en ese sentido a continuación se indican las cantidades asignadas en cada zona de instalación, empezando con el monto a invertir, el número de clientes esperados en cada zona, los dispositivos disponibles para cada zona y la política de instalar al menos un 30\% de los clientes por cada servicio los valores en cada sector.

\section{Esmeraldas Inversión $\quad$ \# clientes Cable Fibra Política de instalación}

\begin{tabular}{|l|ccccc|}
\hline Zona 1 & $\$ 10.000$ & 500 & 228 & 120 & $30 \%$ \\
Zona 2 & $\$ 7.000$ & 400 & 210 & 90 & $30 \%$ \\
Zona 3 & $\$ 8.000$ & 400 & 160 & 160 & $30 \%$ \\
Zona 4 & $\$ 6.000$ & 300 & 184 & 46 & $30 \%$ \\
Zona 5 & $\$ 8.000$ & 350 & 218 & 72 & $30 \%$
\end{tabular}

Según información entregada por la empresa, el total de postes asignados es de 375, lo cual daría un total de 2.625 clientes que pueden ser ubicados como capacidad máxima de instalación, se considera un total de 1950 clientes. Se considera por parte de la empresa un 74\% de servicio de cobertura en la población como referencia para realizar los cálculos de asignación. Se espera instalar en cada zona al menos un $30 \%$ del servicio sea este de fibra o de cable. El modelo matemático en cada zona contemplar maximizar el número de unidades, cumpliendo las restricciones de dispositivos disponibles, el menor \# de unidades en cada sector y un estimado del $74 \%$ de clientes en total del área sin cobertura. En la zona 1 se estima que en cada poste 5 clientes pueden requerir el servicio de cable y 2 clientes el servicio de fibra.

\footnotetext{
\begin{tabular}{l|l}
310 & Vol. 5, núm. 1, enero 2019, pp. 299-325 \\
Dennis H. Zambrano-Silva; Luis E. Arguello-Cortez; Javier Domínguez De La Torre; Eladio
\end{tabular} Bautista-Chalar
} 


\section{Zona 1}

Max. $=68 \times 1+100 \times 2$

\section{Sujeto A:}

$$
\begin{array}{ll}
73 \times 1+120 \times 2<=10.000 & \text { Inversión de Instalación } \\
5 \times 1+2 \times 2<=500 & \text { Asignación de servicio } \\
x 1<=228 & \text { Suministro (cantidad de dispositivo X1) } \\
\mathrm{x} 2<=120 & \text { Suministro (cantidad de dispositivos X2) }
\end{array}
$$
$\mathrm{x} 1>=70$
Mínimo instalar x1
$\mathrm{x} 2>=40$
Mínimo instalar x2

$\mathrm{X} 1, \mathrm{X} 2>=\mathbf{0}$

\section{Área 2}

$\operatorname{Max}=68 \times 1+100 \times 2$

\section{Sujeto A:}

$73 \times 1+120 \times 2<=7000$ Inversión de Instalación

$$
\begin{array}{ll}
5 \mathrm{x} 1+2 \times 2<=400 & \text { Asignación de servicio } \\
\mathrm{x} 1<=210 & \text { Suministro (cantidad de dispositivo X1) } \\
\mathrm{x} 2<=90 & \text { Suministro (cantidad de dispositivos X2) }
\end{array}
$$
$\mathrm{x} 1>=70$
Mínimo instalar x1
$\mathrm{x} 2>=30$
Mínimo instalar x2

$\mathrm{X} 1, \mathrm{X} 2>=\mathbf{0}$

\section{Área 3}


$\operatorname{Max}=68 \times 1+100 x 2$

\section{Sujeto A:}

$73 \times 1+120 \times 2<=8.000$ Inversión de Instalación

$$
\begin{array}{ll}
4 \times 1+3 \times 2<=400 & \text { Asignación de servicio } \\
x 1<=160 & \text { Suministro (cantidad de dispositivo X1) } \\
\text { x2 }<=160 & \text { Suministro (cantidad de dispositivos X2) }
\end{array}
$$
$\mathrm{x} 1>=50$
Mínimo instalar x1
$\mathrm{x} 2>=50$
Mínimo instalar x2

$\mathrm{X} 1, \mathrm{X} 2>=\mathbf{0}$

\section{Área 4}

$\operatorname{Max}=68 x 1+100 x 2$

\section{Sujeto A:}

$73 \times 1+120 \times 2<=6.000$ Inversión de Instalación

$$
\begin{array}{ll}
6 \mathrm{x} 1+1 \times 2<=300 & \text { Asignación de servicio } \\
\mathrm{x} 1<=184 & \text { Suministro (cantidad de dispositivo X1) } \\
\mathrm{x} 2<=46 & \text { Suministro (cantidad de dispositivos X2) }
\end{array}
$$
$\mathrm{x} 1>=60$
Mínimo instalar x1
$\mathrm{x} 2>=15$
Mínimo instalar x2

$\mathrm{X} 1, \mathrm{X} 2>=\mathbf{0}$

\section{Área 5}

$\operatorname{Max}=68 \times 1+100 x 2$

\section{Sujeto A:}

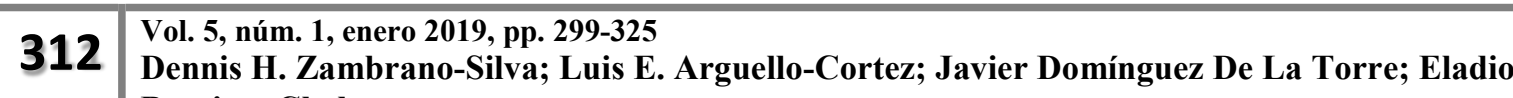
Bautista-Chalar
} 
$73 \times 1+120 \times 2<=8.000$ Inversión de Instalación

$$
\begin{array}{ll}
6 \mathrm{x} 1+1 \mathrm{x} 2<=350 & \text { Asignación de servicio } \\
\mathrm{x} 1<=218 & \text { Suministro (cantidad de dispositivo X1) } \\
\mathrm{x} 2<=72 & \text { Suministro (cantidad de dispositivos X2) }
\end{array}
$$

$\mathrm{x} 1>=70 \quad$ Mínimo instalar $\mathrm{x} 1$

x2 $>=25 \quad$ Mínimo instalar x2

$\mathrm{X} 1, \mathrm{X} 2>=\mathbf{0}$

\section{Análisis de resultados mediante el modelo de programación lineal}

Se utiliza El Solver en cada una de las áreas descritas y se obtienen los siguientes resultados correspondientes a las áreas que fueron determinadas para maximizar el número de dispositivos de acuerdo con la cantidad de dinero con que se cuenta para la inversión, los resultados se presentan en la Tabla $2-3-4-5-6$ respectivamente. 
Dom. Cien., ISSN: 2477-8818

Vol. 5, núm.1. ene., 2019, pp. 299-325

Programación lineal y software para cobertura aplicado a la instalación del servicio de internet en la empresa Solintel S.A

\section{Tabla 2: Modelo Planteado de la zona 1 Resuelto}

\begin{tabular}{|c|c|c|c|c|c|c|}
\hline \multirow{2}{*}{\multicolumn{2}{|c|}{$\begin{array}{l}\text { ZONA } 1 \\
\text { UNIDADES A INSTALAR }\end{array}$}} & \multicolumn{2}{|c|}{$\mathrm{x} 1 \quad \mathrm{x} 2$} & \multicolumn{3}{|c|}{$\mathrm{Z}=$ MAXIMIZAR $\#$ DE INSTALACIONES } \\
\hline & & 71.2 & 40.0 & $\$ 8,844$ & & \\
\hline \multicolumn{2}{|l|}{ Costo Dispositivo } & $\$ 68$ & \multicolumn{4}{|l|}{$\$ 100$} \\
\hline & & & & \multicolumn{2}{|l|}{ Recursos consumidos } & Restricciones \\
\hline Inversión instalación & R1 & $\$ 73$ & $\$ 120$ & $\$ 10,000$ & $<=$ & $\$ 10,000$ \\
\hline Asignac. Servicio poste & $\mathrm{R} 2$ & 5 & 2 & 436.2 & $<=$ & 500 \\
\hline \# dispositivos $\mathrm{x} 1$ & R3 & 1 & 0 & 71.2 & $<=$ & 228 \\
\hline \# dispositivos $\mathrm{x} 2$ & R4 & 0 & 1 & 40.0 & $<=$ & 120 \\
\hline Mínimo instalar x1 & R6 & 1 & 0 & 71.2 & $>=$ & 70 \\
\hline Mínimo instalar x2 & R5 & 0 & 1 & 40.0 & $>=$ & 40 \\
\hline
\end{tabular}

Fuente: Propia

Tabla 3: Modelo Planteado de la zona 2 Resuelto

\begin{tabular}{|c|c|c|c|c|c|c|}
\hline \multirow{2}{*}{\multicolumn{2}{|c|}{$\begin{array}{l}\text { ZONA } 2 \\
\text { UNIDADES A INSTALAR }\end{array}$}} & $\mathrm{x} 1$ & $\mathrm{x} 2$ & \multicolumn{3}{|c|}{$Z$ = MAXIMIZAR \# DE INSTALACIONES } \\
\hline & & 70.0 & 15.8 & $\$ 6,335$ & & \\
\hline \multicolumn{2}{|l|}{ Costo dispositivo } & $\$ 68$ & \multicolumn{4}{|l|}{$\$ 100$} \\
\hline & & & & \multicolumn{2}{|l|}{ Recursos consumidos } & Restricciones \\
\hline Inversión instalación & $\mathrm{R} 1$ & $\$ 73$ & $\$ 120$ & $\$ 7,000$ & $<=$ & $\$ 7,000$ \\
\hline Asignac. Servicio poste & $\mathrm{R} 2$ & 5 & 2 & 381.5 & $<=$ & 400 \\
\hline \# dispositivos $\mathrm{x} 1$ & $\mathrm{R} 3$ & 1 & 0 & 70.0 & $<=$ & 210 \\
\hline \# dispositivos $\mathrm{x} 2$ & R4 & 0 & 1 & 15.8 & $<=$ & 90 \\
\hline Mínimo instalar x1 & R6 & 1 & 0 & 70.0 & $>=$ & 70 \\
\hline Mínimo instalar x2 & R5 & 0 & 1 & 15.8 & $>=$ & 30 \\
\hline
\end{tabular}

Fuente: Propia 
Dom. Cien., ISSN: 2477-8818

Vol. 5, núm.1. ene., 2019, pp. 299-325

Programación lineal y software para cobertura aplicado a la instalación del servicio de internet en la empresa Solintel

Tabla 4: Modelo Planteado de la zona 3 Resuelto

\begin{tabular}{|c|c|c|c|c|c|c|}
\hline \multirow{2}{*}{\multicolumn{2}{|c|}{$\begin{array}{l}\text { ZONA } 3 \\
\text { UNIDADES A INSTALAR }\end{array}$}} & \multirow{2}{*}{$\begin{array}{l}\mathrm{x} 1 \\
50.0\end{array}$} & \multirow{2}{*}{$\frac{x 2}{36.3}$} & \multicolumn{3}{|c|}{$\mathrm{Z}=$ MAXIMIZAR \# DE INSTALACIONES } \\
\hline & & & & $\$ 7,025$ & & \\
\hline \multirow{2}{*}{\multicolumn{2}{|c|}{ Costo dispositivo }} & $\$ 68$ & \multirow[t]{2}{*}{$\$ 100$} & & & \\
\hline & & & & \multicolumn{2}{|l|}{ Recursos consumidos } & Restricciones \\
\hline Inversión instalación & $\mathrm{R} 1$ & $\$ 73$ & $\$ 120$ & $\$ 8,000$ & $<=$ & $\$ 8,000$ \\
\hline Asignac. Servicio poste & $\mathrm{R} 2$ & 4 & 3 & 308.8 & $<=$ & 400 \\
\hline \# dispositivos $\mathrm{x} 1$ & R3 & 1 & 0 & 50.0 & $<=$ & 160 \\
\hline$\#$ dispositivos $\mathrm{x} 2$ & R4 & 0 & 1 & 36.3 & $<=$ & 160 \\
\hline Mínimo instalar x1 & R6 & 1 & 0 & 50.0 & $>=$ & 50 \\
\hline Mínimo instalar $\mathrm{x} 2$ & R5 & 0 & 1 & 36.3 & $>=$ & 50 \\
\hline
\end{tabular}

Fuente: Propia

Tabla 5: Modelo Planteado de la zona 4 Resuelto

\begin{tabular}{|c|c|c|c|c|c|c|}
\hline \multirow{2}{*}{\multicolumn{2}{|c|}{$\begin{array}{l}\text { ZONA } 4 \\
\text { UNIDADES A INSTALAR }\end{array}$}} & \multirow{2}{*}{$\begin{array}{l}\mathrm{x} 1 \\
47.5\end{array}$} & \multirow{2}{*}{$\begin{array}{l}\mathrm{x} 2 \\
15.0\end{array}$} & \multicolumn{3}{|c|}{$\mathrm{Z}=$ MAXIMIZAR \# DE INSTALACIONES } \\
\hline & & & & $\$ 4,730$ & & \\
\hline \multicolumn{2}{|l|}{ Costo dispositivo } & $\$ 68$ & \multicolumn{4}{|l|}{$\$ 100$} \\
\hline & & & & \multicolumn{2}{|l|}{ Recursos consumidos } & Restricciones \\
\hline Inversión instalación & $\mathrm{R} 1$ & $\$ 73$ & $\$ 120$ & $\$ 5,268$ & $<=$ & $\$ 6,000$ \\
\hline Asignac. Servicio poste & $\mathrm{R} 2$ & 6 & 1 & 300.0 & $<=$ & 300 \\
\hline \# dispositivos $\mathrm{x} 1$ & R3 & 1 & 0 & 47.5 & $<=$ & 184 \\
\hline \# dispositivos $\mathrm{x} 2$ & $\mathrm{R} 4$ & 0 & 1 & 15.0 & $<=$ & 46 \\
\hline Mínimo instalar $\mathrm{x} 1$ & R6 & 1 & 0 & 47.5 & $>=$ & 60 \\
\hline Mínimo instalar x2 & $\mathrm{R} 5$ & 0 & 1 & 15.0 & $>=$ & 15 \\
\hline
\end{tabular}

Fuente: Propia

315 Vol. 5, núm. 1, enero 2019, pp. 299-325

Dennis H. Zambrano-Silva; Luis E. Arguello-Cortez; Javier Domínguez De La Torre; Eladio

Bautista-Chalar 
Dom. Cien., ISSN: 2477-8818

Vol. 5, núm.1. ene., 2019, pp. 299-325

\section{Tabla 6: Modelo Planteado de la zona 5 Resuelto}

\begin{tabular}{|c|c|c|c|c|c|c|}
\hline \multirow{2}{*}{\multicolumn{2}{|c|}{$\begin{array}{l}\text { ZONA } 5 \\
\text { UNIDADES A INSTALAR }\end{array}$}} & \multicolumn{2}{|r|}{$\mathrm{x} 2$} & \multicolumn{3}{|c|}{$\mathrm{Z}=$ MAXIMIZAR \# DE INSTALACIONES } \\
\hline & & 54.2 & 25.0 & $\$ 6,183$ & & \\
\hline \multirow{2}{*}{\multicolumn{2}{|c|}{ Costo dispositivo }} & $\$ 68$ & \multirow[t]{2}{*}{$\$ 100$} & & & \\
\hline & & & & \multicolumn{2}{|l|}{ Recursos consumidos } & Restricciones \\
\hline Inversión instalación & $\mathrm{R} 1$ & $\$ 73$ & $\$ 120$ & $\$ 6,954$ & $<=$ & $\$ 8,000$ \\
\hline Asignac. Servicio poste & $\mathrm{R} 2$ & 6 & 1 & 350.0 & $<=$ & 350 \\
\hline \# dispositivos $\mathrm{x} 1$ & R3 & 1 & 0 & 54.2 & $<=$ & 218 \\
\hline \# dispositivos $\mathrm{x} 2$ & $\mathrm{R} 4$ & 0 & 1 & 25.0 & $<=$ & 72 \\
\hline Mínimo instalar x1 & R6 & 1 & 0 & 54.2 & $>=$ & 70 \\
\hline Mínimo instalar x2 & R5 & 0 & 1 & 25.0 & $>=$ & 25 \\
\hline
\end{tabular}

Fuente: Propia

A través de los modelos matemáticos planteados logramos maximizar recursos, dando como resultado valores monetarios inferiores a los valores asignados como presupuesto de inversión de cada zona. Se observa que en los modelos se cumplen todas las restricciones de la zona 1 pero en los demás cálculos las ecuaciones en donde el lado derecho de la ecuación debe ser superior al límite indicado en la restricción, no se cumplen pues se excede en el presupuesto zonal para ese tipo de variable de decisión. Por lo tanto, la política de instalación del 30\% del tipo de dispositivos en cada área no puede cumplirse sin afectar la optimización en la instalación de los equipos. El cuadro siguiente resume los resultados obtenidos 


\section{Tabla 7 Resumen Programación lineal}

\begin{tabular}{l|lll|} 
Zona & Cable UTP & Fibra & Inversión \\
\hline Zona 1 & 71.2 & 40.0 & $\$ 8.844$ \\
Zona 2 & 70.0 & 15.8 & $\$ 6.335$ \\
Zona 3 & 50.0 & 36.3 & $\$ 7.025$ \\
Zona 4 & 47.5 & 15.0 & $\$ 4.730$ \\
Zona 5 & 54.2 & 25.0 & $\$ 6.183$
\end{tabular}

Una vez realizada nuestra maximización de recursos y un mapeo general de los puntos de acceso o red en cobertura. A continuación, debemos localizar los dispositivos intermedios para la conectividad de la red.

Se determinar las áreas a cubrir por X1 hasta X5 las 5 zonas a cubrir, cada una de estas áreas donde se requiere asignar el personal de trabajo cuenta con alrededor de 15 manzanas en los lugares más amplios y alrededor de 10 manzanas en las zonas más pequeñas para ello se asignan grupos o cuadrillas que estarán a cargo de distribuir el servicio de internet a los lugares con déficit en cobertura. Si se considera el costo de la instalación por cuadrilla y el tiempo empleado es posible por medio del método húngaro realizar la mejor asignación.

$$
\begin{aligned}
& \mathrm{X} 1=\text { Área } 1 \text { a Cubrir } \\
& \mathrm{X} 2=\text { Área } 2 \text { a Cubrir } \\
& \mathrm{X} 3=\text { Área } 3 \text { a Cubrir } \\
& \mathrm{X} 4=\text { Área } 4 \text { a Cubrir } \\
& \mathrm{X} 5=\text { Área } 5 \text { a Cubrir }
\end{aligned}
$$

\footnotetext{
\begin{tabular}{l|l}
317 & Vol. 5, núm. 1, enero 2019, pp. 299-325 \\
Dennis H. Zambrano-Silva; Luis E. Arguello-Cortez; Javier Domínguez De La Torre; Eladio
\end{tabular} 


\section{Grafica 3 Áreas a cubrir con carencia de cobertura}

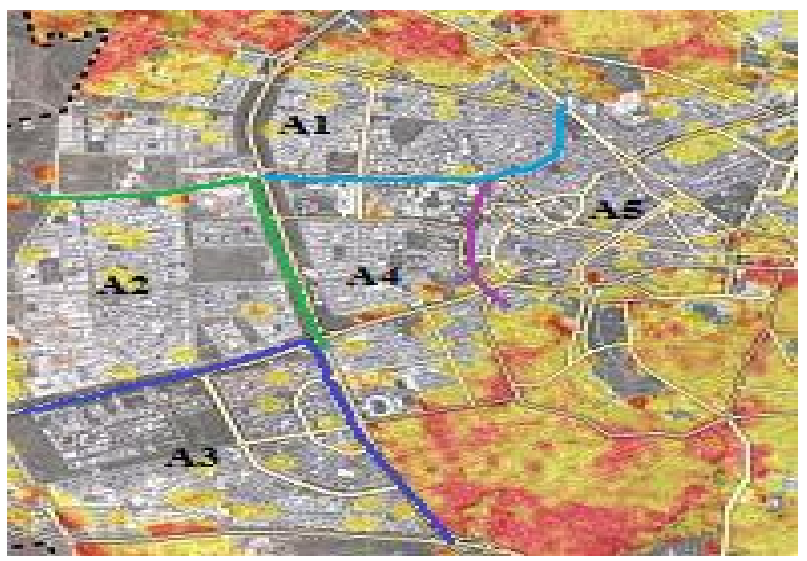

Fuente: Propia

Después de visualizar los puntos donde hay carencia de red y dispositivos por parte del proveedor de servicios de internet SOLINTEL S.A se procede a indicar el problema a solucionar y asignar sus respectivos costos que se requieren para cubrir por área, donde cada uno de estos costos incluyen.

- Transporte de Dispositivos

- Costo de Mano de obra por cuadrilla

- Costos de Instalación

Una vez implementada las metodologías respectivas y software correspondientes donde mediante su análisis se llegaron a cubrir las áreas o sectores en los cuales había un déficit de cobertura. En base al análisis de monitoreo y maximización de las áreas donde la empresa tenía inconveniente pudimos apreciar mediante un análisis informático con la ayuda de software que todavía existían áreas en las cuales la potencia de la señal no era del todo optima, en cuando a velocidad y accesibilidad provocando un problema a grupos de personas y empresas. 
Al surgir un nuevo inconveniente en las mismas áreas de trabajo reagrupamos al personal que se encarga de realizar el servicio de instalación de dispositivos de este modo se implementaron nuevas asignaciones permitiendo que se llegue a optimizar y mejorar la cobertura en aquellas zonas donde la potencia de la red es Regular y Buena llegando a obtener no solo una mejora en cuanto a ancho de banda sino también una mejora de Velocidad en cuanto a carga y descarga de datos que uno o varios usuarios requieran.

Para determinar este modelo fue necesaria una nueva asignación de variables que nos indiquen que va a realizar cada una de las mismas permitiendo que se realice un costo mínimo por asignación. Para establecer esta mejora y poder aplicar el modelo matemático húngaro se llegó analizar todas las maneras posibles para optimizar recursos o mejora de dispositivos por lo cual se optó por un software para dispositivos finales como son laptop, smartphone, Tablet, etc. que permite medir la potencia y la cobertura de uno o varios dispositivos de red logrando saber exactamente cuál es el ancho de banda del dispositivo y la distancia que puede llegar a cubrir el mismo.

\section{Análisis de resultados en Acrylic wifi mediante modelo de programación lineal para abarcar soluciones de cobertura.}

El monitoreo que se realiza mediante este software es factible y sencillo ya que el personal encargado tendrá conocimiento acerca de la herramienta permitiendo que se mejore el funcionamiento de la accesibilidad a la red mediante los dispositivos intermediarios.

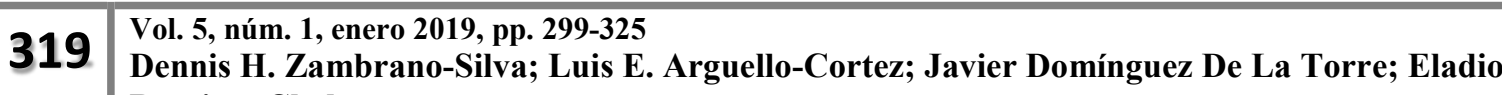
Bautista-Chalar
} 


\section{Medidor de potencia de redes WIFI- OPEN SIGNAL}

\section{Grafica 5: Interfaz Open Signal}

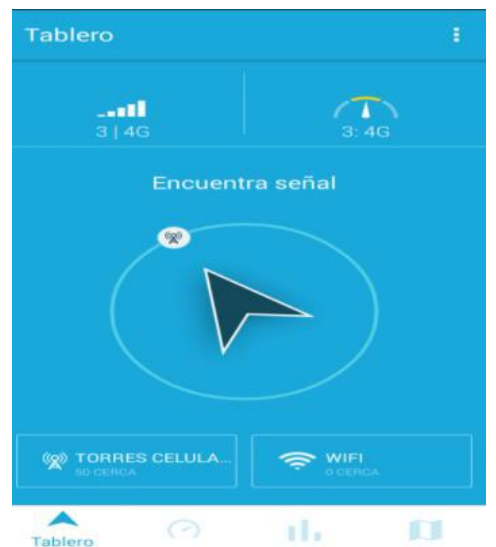

Requisitos de la App:

Espacio de Memoria que ocupa: 3,26 MB

Versión Android para celulares: 4.4 en

adelante

Fuente: Google Play

\section{Elaborado por: Open Signal}

Las aplicaciones para dispositivos móviles son consideradas una necesidad en los tiempos actuales ya que con ellas se fomentan grandes procesos logrando una mayor efectividad a los servicios o prestaciones como tal.

El principal objetivo de implementar o dar a conocer el software utilizado para medir potencia de señal que hay en los dispositivos móviles es para optimizar el alcance de la señal como tal mediante el cambio de dispositivos o una asignación de canales en la cual uno o varios usuarios puedan tener mayor facilidad al conectarse a la red.

Uno de los beneficios que nos brinda el software como tal es una aplicación OFF-LINE, por lo tanto el uso del servicio de internet es innecesario al momento de buscar analizar y mejorar la cobertura en determinadas áreas, para tener una mejor perspectiva daremos a conocer sus características a continuación: 
- Totalmente Gratis

- Analiza la velocidad de tu conexión

- Cambia las tarjetas SIM y compara la cobertura de operadores diferentes

- Traza un mapa de tu cobertura móvil y WiFi.

\section{Análisis de los dispositivos de red mediante el Software Open Signal}

El personal encargado de verificar la potencia y distancia de los dispositivos de red deberán tener la aplicación en sus dispositivos móviles para facilitar el trabajo al momento de realizar un test de velocidad y potencia en el área que se les haya sido asignada, permitiéndoles tener datos precisos del o los dispositivos monitoreados, luego se encargará de asignar un costo especifico el cual contendrá los cambios a realizarse para mejorar la potencia de la señal en cuanto hablamos de cobertura en caso de ser necesario como se detalla a continuación:

Cambio y movilización de los Dispositivos de Red

- Cableado en mal estado

- Cajetín o Modem mal configurado

- Costo de Instalación de cable UTP o Fibra Óptica

- El tipo de dispositivo utilizar 


\section{Grafica 7: Comprobación de Potencia y Velocidad}

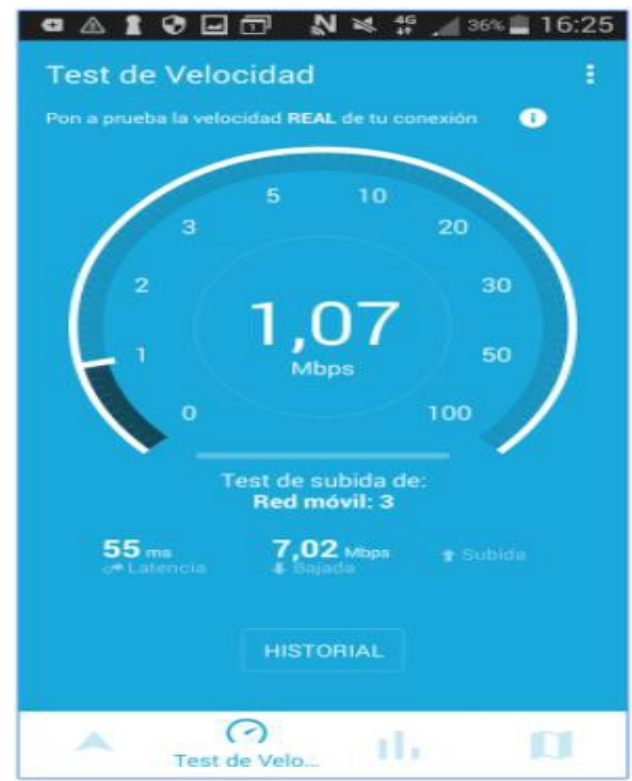

Fuente: Google App Play

Elaborado por: Open Signal

Las variables que se llegaran a utilizar para realizar el modelo húngaro tendrán una asignación que será por área, y su vez otra asignación que será por cuadrilla a lo que respecta al personal técnico encargado de cubrir una de estas áreas, para que esto sea posible cada sector tiene un costo asignado y variables que se detallara a continuación:

$$
\begin{array}{ll}
\text { X1 }=\text { Área } 1 \text { a Cubrir } & \mathrm{C} 1=\text { Cuadrilla } 1 \\
\text { X2 = Área 2 a Cubrir } & \mathrm{C} 2=\text { Cuadrilla 2 } \\
\mathrm{X} 3=\text { Área 3 a Cubrir } & \mathrm{C} 3=\text { Cuadrilla } 3 \\
\mathrm{X} 4=\text { Área } 4 \text { a Cubrir } & \mathrm{C} 4=\text { Cuadrilla } 4 \\
\text { X5 }=\text { Área } 5 \text { a Cubrir } & \mathrm{C} 5=\text { Cuadrilla } 5
\end{array}
$$




\section{Resultados.}

Con la aplicación de la programación lineal las variables de decisión se subordinan al presupuesto en cada área de instalación a un mínimo costo de inversión. Es viable la utilización de herramientas como el Solver para el cálculo y la aplicación de diferentes tipos de software como el Open signal a bajo costo se obtienen buenos resultados con respecto a evaluar la cobertura, entendiendo que mediante el uso de modelos lineales se obtienen una solución factible generándonos una maximización de recursos y obtención de dispositivos de red por cada grupo de usuarios o empresas.

\section{Conclusiones.}

La programación lineal permite proporcionar métodos particulares en lo cual el objetivo como tal es brindar una gran ayuda de dar futuras estrategias de desarrollo y mejora de una empresa.

Cabe acotar que entregamos un modelo estándar, y muy eficiente con una facilidad de usar, desde un punto de vista práctico y consigo algunas virtudes mediante los programas lineales y no lineales.

Todo resultado de asignación que se generan de cada área a cubrir respecto a cobertura tiene que estar asociados con el software de Acrylic Heat Maps y Open Signal ya que mediante este software permite saber qué área a cada técnico o cuadrilla en general le corresponde.

El software se mostrará el dispositivo de red el cual muestre déficit en cobertura y a su vez otro indicará de manera general la visualización de las áreas permitiendo que se logre una optimización de dispositivos y maximización de recursos.

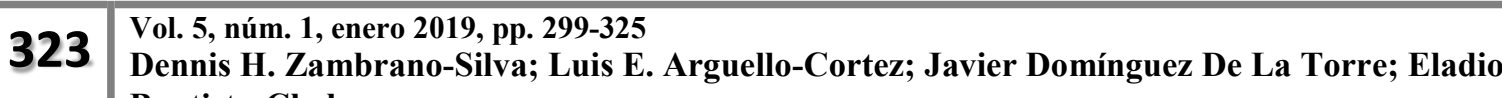


El modelo de asignación Húngaro resulta muy útil al momento de asignar las cuadrillas de instalación de los diferentes equipos o empresas de instalación contratadas. Es posible asignar costos a cada asignación, para resolver de manera efectiva con el menor recurso posible y disponible. (Szanyi, 2004). Es decir, el modelo húngaro permite que las asignaciones o labores determinadas que se realizan en ciertas áreas sean más eficientes para un empleado o departamento como tal (López Reyes, 2016). Con ello la asignación permite la minimización del costo y solucionar los problemas de cobertura (Lewandowski, 2015).

\section{Bibliografía.}

Calderón, L. A. F., Ocampo, E. M. T., \& Echeverry, M. G. (2012). Diseño de redes de logística inversa: una revisión del estado del arte y aplicación práctica. Ciencia E Ingeniería Neogranadina, 22(2), 153-127.

Comunicaci, E. N. R. D. E., Final, I., Estudio, C. D. E., Unidad, P., Tema, E., Para, E., ... Militar, F. (2016). Pontificia universidad católica del ecuador.

Evans, D. (2011). Internet de las cosas Internet de las cosas Cómo la próxima evolución de Internet lo cambia todo.

Guédez Fernández, C. (2011). Programación Lineal e Ingeniería Industrial: una Aproximación al Estado del Arte. Ingeniería Industrial. Actualidad Y Nuevas Tendencias, II(6), 61-78. Retrieved from http://www.redalyc.org/pdf/2150/215021914005.pdf

Kalenatic, D. (2009). M odelo de a m pliación de la capacidad productiva. Revista de Ingenieria $U D$.

Lewandowski, C. M. (2015). Modelo De Programación Lineal Aplicado a La Red Logística De Una Empresa Del Sector Plástico. The Effects of Brief Mindfulness Intervention on Acute Pain Experience: An Examination of Individual Difference, 1. https://doi.org/10.1017/CBO9781107415324.004

López Reyes, D. (2016). El método húngaro de asignación: aplicaciones. Retrieved from https://idus.us.es/xmlui/handle/11441/43823

Ospina, L., Rodas, P., \& Botero, M. (2008). Modelo de programación para integrar producción, inventario y ventas en empresas industriales. Scientia Et Technica, XIV(40), 101-104. Retrieved from http://www.redalyc.org/articulo.oa?id=84920454019 
Programming, L., Programming, L., Value, O., \& Prices, D. (2009). LA PROGRAMACIÓN LINEAL APLICACIÓN DE LA PEQUEÑAS Y MEDIANAS EMPRESAS Jorge Alvarado Boirivant *, 88(1), 89-105.

Raña K, J., Ferrer O, J.-C., \& Bedregal G, P. (2007). A model for resource allocation in primary health care. Revista Medica de Chile, 135(1), 54-62. https://doi.org//S003498872007000100008

Redes, A. D. E., Mediante, D. E. C., Probabilisticos, M. N. O., Presenta, M. Q. U. E., Francisco, J., \& Guadix, H. (n.d.). No Title.

Szanyi, M. (2004). El modelo de desarrollo húngaro basado en la IDE. Boletín Económico de ICE, Información Comercial Española, ISSN 0214-8307, No 2797, 2004, Págs. 47-58, (2797), 4758. 